\title{
La investigación sobre nuestros alumnos, en pro de una mayor eficacia en la enseñanza universitaria
}

\section{Paul Sander}

Departamento de Psicología, Universidad de Wales Institute, Cardiff

\section{Reino Unido}

psander@uwic.ac.uk

\begin{abstract}
Resumen
Para proporcionar una educación eficiente y eficaz, es imprescindible que los profesores conozcan las características de sus alumnos como aprendices. Cuando las clases son pequeñas, los métodos informales pueden ser suficientes; sin embargo, el clima actual de la Educación Superior promociona una mayor participación y diversidad de alumnos, como es debido, y esto produce clases más numerosas y menos homogéneas, ya que no ha habido un aumento concomitante de recursos. Por lo tanto, puede ser necesario incorporar métodos más formales para comprender a los alumnos en su papel de aprendices. Los métodos de encuesta serían una estrategia que lo facilita. Éstos pueden emplearse para que los profesores comprendan las expectativas de los alumnos en cuanto a la enseñanza, el aprendizaje y la evaluación; sus concepciones del aprendizaje; sus creencias epistemológicas; y sus capacidades de reflexión. La información adquirida a partir de tales encuestas puede permitir que los profesores construyan ambientes de aprendizaje más eficaces para sus estudiantes numerosos y diversos.
\end{abstract}

Palabras clave: Información de perfil; investigación empírica, expectativas; confianza 


\section{Introducción}

Es necesario conocer y comprender a los nuevos estudiantes porque la eficacia educativa depende en parte de que los profesores puedan establecer un diálogo de doble sentido en el aprendizaje (Laurillard, 1993; Scott, 1999). Las clases numerosas normalmente hacen imposible la aplicación de estrategias conocidas por los profesores, las cuales ayudarían a comprender el punto de partida de cada alumno en su fase actual de la educación. Al mismo tiempo, la mayor diversidad del alumnado hace aún más imprescindible que los profesores comprendan no sólo los conocimientos y habilidades de sus alumnos al empezar, sino comprenderlos también a ellos como aprendices. En breve, los profesores deben comprender las concepciones y percepciones de aprendizaje de sus alumnos.

Para comprender a los estudiantes como aprendices, empezamos con el primer apartado, "La educación superior hoy en día", donde consideramos factores relevantes de trasfondo personal. En el segundo apartado, "La enseñanza universitaria hoy en día", se argumenta que, frente a los recursos reducidos y una mayor diversidad del alumnado, especialmente en el sector de las nuevas universidades, se exige cada vez más que las universidades proporcionen ambientes de aprendizaje estimulantes y eficaces para sus alumnos. Se supone que los licenciados tienen una gama de habilidades que les permite rendir con mayor eficacia en el lugar de trabajo, así produciendo un mayor valor por el dinero que se dedica a la enseñanza superior. En esencia, bien se puede esperar más de un alumnado más diverso, al mismo tiempo que se espera una mayor eficacia de las universidades (Eurydice, 2000).

Un posible método para afrontar este reto se describe en el tercer apartado, "Averiguar más sobre los estudiantes", y se centra en las metodologías de encuesta para grandes grupos de estudiantes. Este argumento encuentra apoyo en la investigación empírica sobre los estudiantes universitarios, así sugiriéndolo como estrategia provechosa.

\section{La educación superior hoy en día}

La educación superior se está transformando en toda Europa (Field, 2002). Se ha incrementado notablemente el número de alumnos que, al acabar la educación secundaria, siguen con estudios universitarios. Esto se está promoviendo al habernos cambiado de una sociedad basada en la producción a ser una sociedad basada en el conocimiento, y también para 
fomentar la estabilidad social característica de las naciones prósperas y en paz (Eurydice, 2000).

La participación en la Educación Superior (ES) del Reino Unido se ha incrementado hasta el punto que más del $40 \%$ de los que terminan su educación secundaria emprenden una educación universitaria, frente al 15\% que lo hacían hace una década (Biggs, 1999). Davis (2003) y Winn (2002) proporcionan una perspectiva general de los cambios en el sector ES del Reino Unido. Asimismo, el alumnado matriculado en las universidades de España se ha duplicado en el período de 1983/4 a 2001/2 (Ministerio de Educación, Cultura y Deporte, España), pero los gastos para la educación superior en España siguen por debajo de la media de los países de la OCDE (Mora, 1999).

En el Reino Unido, si el incremento de estudiantes se hubiera correspondido con incrementos proporcionales del número de profesores y de otros recursos necesarios, entonces el tamaño de las clases y el trabajo de los profesores habrían seguido igual. La National Association for Teachers in Further and Higher Education (asociación nacional para profesores de la educación superior) es uno de los dos sindicatos principales que aboga por los profesores académicos del Reino Unido, y éste ha llamado la atención a los problemas que surgen en la ES al reclutar más estudiantes y una mayor variedad de estudiantes, sin proveer recursos adicionales adecuados (Natfhe, referencia de Internet). Mientras el debate sobre el tamaño de clases en la educación no-universitaria se preocupa por mantener un número de estudiantes inferior a 20 (Bell, 1998; Hart, 1999), la Natfhe expresa preocupación de que, si las clases magistrales llegan a tener 200 estudiantes o más, hay poca oportunidad para un seguimiento de diálogo o ayuda individual (Natfhe, pág. 2). La desigual distribución de fondos económicos en el sector de ES agrava más aún el problema. Los nuevos Higher Education Institutes (HEIs, o, institutos de educación superior) sirven a más del 50\% de los universitarios del Reino Unido. Un 33\% de sus estudiantes provienen de las clases sociales inferiores, frente al 19\% en los antiguos HEIs, sin embargo reciben sólo la quinta parte de fondos económicos por alumno (pág. 4). El aumento de diversidad estudiantil ocurre sobre todo en las universidades que menos fondos reciben por estudiante. Estas universidades también admiten más estudiantes de los estratos sociales, étnicos y de edad menos representados. Para estos estudiantes es más probable que necesiten ayuda adicional, lo que es más difícil ofrecerles si las clases son numerosas. 
Asimismo, también ha aumentado la diversidad del alumnado en España (Ministerio de Educación, Cultura y Deporte, España), igual que en toda Europa. La motivación política de la diversificación del alumnado es que, al proporcionar oportunidades de educación superior para todos, se conduce tanto a la cohesión social como al progreso cultural. También hace falta una población activa altamente cualificada para mantener la competitividad en un mercado global (Eurydice, 2000).

Hoy en día la educación superior les cuesta a muchos estudiantes y a sus familias unas sumas importantes de dinero. Hay muchas consecuencias de este aprieto económico, incluido el trabajo a tiempo parcial entre los estudiantes (¿y por tanto el estudio a tiempo parcial?), así como la expectativa razonable de que esta educación tan costosa debe proporcionar valor a cambio del dinero. El efecto final de esta situación es que la educación superior se vuelve a plantear como un servicio, y los estudiantes son clientes de pago (Hill, 1995; Scott, 1999; Thorne y Cuthbert, 1996).

\section{La enseñanza universitaria hoy en día}

La situación actual es que las universidades, sobre todo las nuevas universidades [británicas], ya tienen un alumnado más diverso. La diversidad del alumnado es también un fenómeno europeo (Eurydice, 2000). Los estudiantes se consideran más bien consumidores de una provisión educativa, más directamente ligada al futuro empleo, por medio de un incremento de cursos de formación profesional (Northedge, 2003). Al mismo tiempo, a las universidades se les exige que consideren la calidad de los ambientes de aprendizaje que proveen para sus estudiantes (Eurydice, 2000). Para considerar la eficacia y la eficiencia de la enseñanza universitaria y el aprendizaje de los estudiantes, se requieren unas medidas del producto final. Un ejemplo ha sido la elaboración articulada de graduateness [el conjunto de cualidades y habilidades que debe reunir el licenciado] dentro del sistema del Reino Unido (HEQC, 1995). Una de las propiedades de graduateness es la de ser aprendiz independiente. Aunque esto no sea un resultado nuevo u original para la educación universitaria, sí es nueva la atención al proceso de convertir el nuevo alumno en aprendiz independiente o autónomo.

Para asegurar la mayor probabilidad de que los licenciados sean aprendices independientes, las universidades han contemplado las experiencias de aprendizaje que se crean para sus estudiantes. Se considera que los siguientes aspectos de ambientes de aprendizaje son eficaces para promover la independencia (UWIC, referencia de Internet): 
- Se centran en el estudiante, frente a centrarse en el profesor.

- Fomentan un enfoque profundo al aprendizaje, obligándole al estudiante que se implique activamente con el tema.

- Requieren que los estudiantes trabajen activamente con el tema, más que quedarse sentados, escuchando, mientras el "experto" habla sobre él.

- Estimulan a los estudiantes a que reflejen sobre el aprendizaje, aprendiendo de lo que les ha salido bien, y considerando lo que no ha funcionado.

- Incluyen a todos los estudiantes (inclusividad), proporcionando métodos de enseñanza y ambientes de aprendizaje que alcancen a todos los estudiantes.

Para fomentar el aprendizaje independiente entre los estudiantes, se han elaborado estrategias específicas de aprendizaje / enseñanza, como el Problem Based Learning [Aprendizaje basado en resolución de problemas] (Schwartz, Mennin y Webb, 2001). También se ha incrementado el uso de estrategias como Personal Development Planning [planificación del desarrollo personal] (Learning Teaching Support Network, 2002), y los estudiantes tienen a su alcance más consejos sobre cómo estudiar.

Éstas, sin embargo, son iniciativas nuevas, no son métodos tradicionales de la enseñanza universitaria. ¿Hasta qué punto fomentan el aprendizaje independiente los métodos de enseñanza habituales? Brown (1993) manifiesta que los métodos de enseñanza se pueden considerar como repartidos en un continuum entre los métodos donde predomina la participación y el control del profesor y los métodos donde predomina la participación y el control del estudiante. La conferencia tradicional es buen ejemplo de un método de enseñanza donde predomina la participación y el control del profesor. A la inversa, las exposiciones estudiantiles son un método de enseñanza donde predomina la participación y el control del estudiante.

Puede que la conferencia haya perdurado en la enseñanza superior porque es relativamente fácil de preparar y de dar, y dado que sólo el mejor $15 \%$ de los estudiantes que terminaban la educación secundaria llegaban a la educación superior, la conferencia era suficientemente eficaz para estos estudiantes inteligentes, interesados, comprometidos y motivados. Ya que se está incrementando el número de estudiantes menos capaces académicamente y quizás inicialmente menos comprometidos, las conferencias pasivas y pesadas pueden ser 
poco eficaces y consiguen poco a la hora de fomentar el aprendizaje independiente en los estudiantes, aunque por supuesto se las puede "enriquecer" (Biggs, 1999) para mayor eficacia.

Con el proyecto de graduateness, se espera de un alumnado universitario más numeroso y más diverso que consigan más que habilidades y conocimientos específicos de las asignaturas. Partiendo de las atribuciones de graduateness, resulta que se requiere una enseñanza dirigida y que apoya, para poder cumplir con la atribución de inclusividad.

\section{Averiguar más sobre los estudiantes}

Para proporcionar una enseñanza dirigida y que apoya, a fin de promover la graduateness, los profesores deben conocer algo de sus estudiantes. Específicamente, los profesores deben conocer de sus alumnos no sólo sus conocimientos específicos de las asignaturas, sino también sus concepciones y percepciones en cuanto a la enseñanza y el aprendizaje. La experiencia colectiva nos sugiere que, al dar clase de manera frecuente a grupos más reducidos, típicos de la educación primaria, este conocimiento viene con relativa facilidad, ocurriendo muchas veces implícitamente, pero, ¿cómo pueden averiguar más sobre sus estudiantes los profesores de grupos de cien o de doscientos alumnos?

Hay por lo menos tres posibilidades. Primero, se puede emplear los métodos tradicionales de sistemas de tutor personal y de dar clase en grupos reducidos, a la medida que lo permite el volumen de trabajo del profesor, y la disponibilidad de otros recursos (Maunder y Harrop, 2003).

Un segundo método depende del uso cada vez más extendido de los ambientes de aprendizaje virtuales, los cuales también pueden facilitar a entablar diálogo y a averiguar más sobre los estudiantes de clases numerosas a través de sus foros electrónicos y las comunicaciones por correo electrónico (Jolliffe, Ritter, y Stevens, 2001).

Un tercer método utiliza herramientas de encuestas para elaborar un cuadro de las virtudes y los defectos de un grupo concreto de estudiantes. Es verdad que esto no proporciona el mismo detalle que se consigue en el aula tradicional, pero puede que sea suficiente. Se les puede encuestar a los estudiantes durante la primera semana de clase para adquirir información sobre, por ejemplo: sus estilos de aprendizaje (Biggs, Kember y Leung 2001); su pensamiento reflexivo (Kember et al, 2000; Leung y Kember, 2003); y sus creencias epistemológicas (Clarebout et al, 2002; Schommer, 1990, 1998). También se puede solicitar las expectativas de los estudiantes (Maunder y Harrop, 2003). 
Sander et al (2000) encuestaron a nuevos estudiantes universitarios sobre sus expectativas de la enseñanza, la evaluación y sus percepciones de un buen profesor. Los hallazgos sobre las expectativas de los estudiantes y sus preferencias por distintos métodos de enseñanza se resumen en la Tabla 1. La investigación en sí se resume en el Cuadro 1.

Tabla 1: Rango y media de rango para las opiniones de los estudiantes sobre métodos de enseñanza universitaria

\begin{tabular}{|l|c|c|c|c|c|c|}
\hline & \multicolumn{2}{|l|}{ Enseñanza que se espera } & \multicolumn{2}{l|}{ Enseñanza que se desea } & \multicolumn{2}{l|}{ Enseñanza no deseada } \\
\hline Rango & $\begin{array}{c}\text { Método de } \\
\text { enseñanza }\end{array}$ & $\begin{array}{c}\text { Media } \\
\text { de } \\
\text { Rango }\end{array}$ & $\begin{array}{c}\text { Método de } \\
\text { enseñanza }\end{array}$ & $\begin{array}{c}\text { Media } \\
\text { de } \\
\text { Rango }\end{array}$ & $\begin{array}{c}\text { Método de } \\
\text { enseñanza }\end{array}$ & $\begin{array}{c}\text { Media } \\
\text { de } \\
\text { Rango }\end{array}$ \\
\hline $1^{\circ}$ & $\begin{array}{c}\text { Conferencia } \\
\text { tradicional }\end{array}$ & 1,69 & $\begin{array}{c}\text { Conferencia } \\
\text { interactiva }\end{array}$ & 1,77 & Dramatización & 1,09 \\
\hline $2^{\circ}$ & $\begin{array}{c}\text { Conferencia } \\
\text { interactiva }\end{array}$ & 1,30 & $\begin{array}{c}\text { Enseñanza } \\
\text { centrada en el } \\
\text { alumno }\end{array}$ & 0,91 & $\begin{array}{c}\text { Conferencia } \\
\text { tradicional }\end{array}$ & 0,96 \\
\hline $3^{\circ}$ & Tutorial & 0,74 & Tutorial & 0,88 & $\begin{array}{c}\text { Exposiciones } \\
\text { estudiantiles }\end{array}$ & 0,86 \\
\hline
\end{tabular}

La Tabla 1 indica que los estudiantes esperan encontrar conferencias interactivas en segundo lugar después de las conferencias tradicionales. La conferencia interactiva destacó como el método de enseñanza más deseada. La preferencia por la conferencia interactiva puede deberse a que éste sea un método familiar de enseñanza para los estudiantes, de su etapa de educación secundaria. También puede ser un método de enseñanza que concuerda con las creencias de los estudiantes sobre el conocimiento y el aprendizaje. Si es así, puede haber forma de ir convirtiendo a tales estudiantes en aprendices autónomos.

Es probable que haya relación entre las preferencias de los estudiantes por ambientes específicos de enseñanza/aprendizaje, sus creencias epistemológicas y sus concepciones del aprendizaje (por ej., Leung y Kember, 2003). Los estudiantes que creen que el conocimiento es fijo y absoluto bien pueden preferir un formato de conferencia, el que les permite ser "receptores" de conocimiento que les transmite un profesor "experto". Para estos estudiantes, una conferencia tradicional funciona sólo mientras puedan seguir la conferencia. Si la conferencia se volviera interactiva, permitiendo que los estudiantes le pidiesen al conferenciante que vaya más despacio, que repita lo que ha dicho, o que dejara más tiempo la transparencia, 
entonces satisfaría sus preferencias. Los estudiantes que creen en el conocimiento como algo fijo y absoluto quizás lo lleven peor en un ambiente de aprendizaje donde a los estudiantes se les pide que trabajen juntos para investigar temas y luego compartan los conocimientos que habían ganado a partir de su investigación (e.g. Sander, 2002). Partiendo de esto, resulta que los estudiantes que esperan que se les "transmitan" los conocimientos, y que éstos luego puedan aprenderse y reproducirse, tendrán dificultades en un ambiente de aprendizaje que no les transmite conocimientos. Asimismo, los estudiantes que buscan adquirir nueva comprensión de un tema trabajando con problemas y cuestiones quizás no les sirva un formato de conferencias.

Es desconcertante observar en la tabla 1 que existen varios desacuerdos con las expectativas de los estudiantes. Por ejemplo, los estudiantes cuentan con las conferencias tradicionales, pero no las desean, tampoco quieren hacer exposiciones, pero muchos cursos las mandan como requisito. Estos desacuerdos nos preocupan porque fácilmente conducen a una enseñanza ineficaz o ineficiente, y tal vez a la desilusión y la falta de interés en los estudiantes (Mann, 2001).

\section{Cuadro 1: Expectativas de los estudiantes universitarios en cuanto a la universidad (Sander, Stevenson, King y Coates, 2000)}

En este estudio se empleó un cuestionario (el USET) diseñado especialmente para explorar las expectativas y preferencias de los universitarios en cuanto a la enseñanza, el aprendizaje y la valoración. Se manejó una muestra por comodidad (convenience sample) de 395 estudiantes del primer curso al inicio de su vida universitaria. Estaban matriculados en carreras de Medicina, Empresariales o Psicología, en una de tres universidades británicas. Una de ellas era una nueva universidad en South Wales. Las otras dos eran universidades tradicionales en East Midlands. La puntuación media de los exámenes A Level fue 27,8 para los estudiantes de medicina, 22,5 para los de empresariales, y 15,0 para los de psicología. Sin embargo, no todos los alumnos habían hecho los exámenes $A$ Level.

Las estadísticas para resumir respuestas de los 395 se computaron asignando una puntuación de 3 a la selección de mayor rango, después 2,1 y 0 para un ítem que no se seleccionó. A partir de estos rangos en orden descendiente, se calcularon las medias y la desviación típica para las respuestas de los 395. Los métodos de enseñanza más seleccionados como esperados, deseados y no deseados se exponen en la tabla 1.

En general, existían más similitudes que diferencias en las expectativas y preferencias de los tres grupos. En concreto, los alumnos esperaban que se les iba a enseñar a través de conferencias tradicionales e interactivas, pero preferían las conferencias interactivas y las actividades en grupo. Sus métodos menos deseados eran la conferencia tradicional, la dramatización y las exposiciones de los estudiantes. Para la valoración del trabajo del curso preferían los 
ensayos, los trabajos de investigación y los problemas / ejercicios. Aunque existía una preferencia general ligeramente a favor de la valoración del trabajo por encima de los exámenes, esto no fue el caso para los tres centros. Puede ser que los estudiantes identificaran los métodos de valoración que conocían. Cuando a los estudiantes se les pidió que clasificaran varias cualidades del buen profesor, eligieron "habilidad para enseñar", seguida por "accesible" como las más importantes.

Sería interesante incluir en esta encuesta los ambientes de aprendizaje virtuales, para ver qué son las opiniones de los estudiantes en cuanto a esta forma de enseñanza cada vez más extendida, la cual "ya forma parte de la vida" (Hartley, 1998, pág. 105).

Si se sabe que los estudiantes tienen expectativas que no concuerdan con lo que les ofrece la universidad, sean específicamente en cuanto a la enseñanza o el aprendizaje, o más generalmente en cuanto a cuestiones del ambiente universitario, se puede responder a estos desacuerdos de dos posibles formas. O se manejan las mismas expectativas, conduciéndolas a lo que les ofrece la universidad, o el curso (p.ej. Hill, 1995), o se puede responder cambiando la oferta para que concuerde con lo que esperan los estudiantes (p.ej. Stevenson, Sander y Naylor, 1996, 1997). No obstante, cambiando la oferta para concordar con las expectativas de los estudiantes no significa que a los estudiantes siempre hay que darles lo que deseen. Por ejemplo, los estudiantes encuentran intimidatorias las exposiciones (Sander y Stevenson, 2002; Stevenson y Sander, 2002), pero éstas pueden ser provechosas (Sander, Sanders y Stevenson, 2002). El Cuadro 2 resume unos hallazgos recientes sobre los efectos de las exposiciones estudiantiles.

\section{Cuadro 2: Hacer Exposiciones: su impacto sobre las percepciones de los estudiantes (Sander y Sanders, 2005)}

La investigación ha mostrado que a los estudiantes no les gustan las exposiciones, sin embargo hay argumentos a su favor (Sander, Sanders y Stevenson, 2002). Este trabajo pretende comprender los efectos que las exposiciones producen en los estudiantes.

Dentro de un marco de investigación-acción, y usando un diseño factorial de $2 \times 2$, se realizaron dos estudios, en uno se valoraron las exposiciones de los estudiantes, en el otro ellos hacían exposiciones sin ser valorados. El factor 1 era Tiempo: principio y final del semestre, factor 2 era condición: antes o después de la exposición. Todos los sujetos rellenaron las escalas Academic Behavioural Confidence (ABC) y Views on Teaching, Learning and Assessment (VTLA) en ambos momentos de tiempo. 
Los estudiantes que hicieron exposiciones con valoración presentaron un incremento general en la $A B C,(\mathrm{p}<0.05)$. No se encontró ningún incremento similar después de las exposiciones no valoradas. En ambos estudios, los estudiantes mostraron un incremento significativo en sus respuestas a los ítems de la $A B C$ que tenían que ver con hablar en público $(\mathrm{p}<0.05)$.

La VTLA descubrió que la experiencia de exposiciones como método de enseñanza puede ayudar a que los estudiantes se sientan más positivos sobre ellas, y que sean más capaces de reconocer sus beneficios. También confirmó que los estudiantes se quedan intimidados ante las exposiciones, y que algunos tienen dudas sobre la eficacia de que les enseñen sus iguales.

Los distintos resultados de los dos estudios pueden verse afectados por la manera de la que se integraron las exposiciones en los módulos en distintos niveles. Sin embargo, parece que la experiencia de las exposiciones puede incrementar la confianza de los estudiantes en sus propias capacidades, aunque es menos probable que cambie su punto de vista ante la perspectiva de hacer exposiciones.

Stevenson, Sander y Naylor (1996), en un trabajo de investigación-acción, demostraron que se podía crear, para estudiantes que cursaban una carrera a distancia, sesiones presenciales más eficaces, recogiendo datos sobre sus preferencias en cuanto a la enseñanza y el aprendizaje a través de cuestionarios. Se emplearon estas preferencias para estructurar un programa de sesiones que se elaboraba a partir de un estilo didáctico, el cual los estudiantes habían indicado que lo preferían antes que los talleres participativos y facilitados donde se empleaban métodos de enseñanza no deseados, como dramatizaciones y exposiciones estudiantiles. Cuando se empleaban métodos de enseñanza no deseados según los cuestionarios, se proporcionaba más apoyo a los estudiantes. Las evaluaciones del programa de sesiones presenciales sugieren que el programa rediseñado les gustaba a más estudiantes durante mayor proporción del tiempo, y a la vez servía como una ayuda para los estudiantes en su transición a ser aprendices independientes. Así los estudiantes pueden llegar a beneficiarse de y a valorar métodos de enseñanza que al principio no preferían. Reconocer, por ejemplo, que a los estudiantes no les gustan las exposiciones estudiantiles, y apoyarles en esa fase, es un ejemplo de que los profesores "se conecten" con los estudiantes.

Este trabajo se resume en el Cuadro 3. 


\section{Cuadro 3: Percepciones estudiantiles sobre el papel del tutor en el aprendizaje a distancia (Stevenson, Sander y Naylor, 1996)}

Esta investigación-acción la realizó un grupo de tutores que impartían el mismo curso de psicología de la Open University, trabajando en la misma región pero en distintos centros, y con clases presenciales eventuales en común. La investigación pretendió explorar las expectativas de estos aprendices a distancia sobre sus clases presenciales aisladas. Se argumenta que esta información sirve para desarrollar un mayor conocimiento de la relación tutor-aprendiz, y su interacción con el proceso de aprendizaje.

El equipo de investigación diseñó un estudio de doble orientación para averiguar las expectativas de los estudiantes. A diez estudiantes se les entrevistó a fondo con un guión de entrevista, mientras a 94 estudiantes se les proporcionó un conjunto similar de preguntas abiertas como cuestionario por correo postal. Se recogieron los datos de las entrevistas y de los cuestionarios antes de la primera clase presencial para todos los alumnos. Al final del curso, se les encuestó a los estudiantes por entrevista y por cuestionario postal, por conocer su evaluación del programa tutorial.

Los datos indicaron que:

\section{A los estudiantes les gustaba...}

- Una variedad de métodos de enseñanza

- Metas y objetivos definidos

- Programa / avisos por adelantado

- Comentarios alentadores sobre sus trabajos realizados

- Oportunidades para preparar los exámenes

A los estudiantes no les gustaba...

- Trabajo en grupo que no llegaba a ninguna parte

- Ser elegidos para contestar una pregunta

- Calificaciones muy indulgentes

- Correcciones pedantes de ortografía y gramática

- Comentarios vagos y generales sobre los trabajos

A los estudiantes les gustaría...

- Que los tutores llegasen preparados

- Que las conferencias se impartieran bien y que hubiera oportunidad para hacer preguntas

- Que los tutores alentasen y apoyasen a los estudiantes

- Que los tutores demostraran buen humor y diálogo en sus correcciones

- Que las clases presenciales estuvieran bien planificadas y útiles, así justificando el esfuerzo necesario para poder asistir a ellas.

Este estudio muestra que el conocimiento de lo que esperan los estudiantes puede proporcionarles a los profesores la oportunidad de considerar cómo se debería adaptar sus estrategias de enseñanza para que funcionasen con más eficacia, sobre todo si éstas distan mucho de las expectativas de sus alumnos. 
Así es que, a pesar del posible avance de una cultura del cliente, no es necesario que la enseñanza superior siempre cumpla la expectativas de los estudiantes (Scott, 1999). Lo primordial es el diseño de ambientes de aprendizaje eficaces, lo cual no tiene por qué ser fácil (Clarebout, Elen, Johnson, y Shaw, 2002; Clarebout, Lowyck y Elen, 2003; Elen y Clarebout, 2002). Como afirma Shuell (1986): "Si los estudiantes han de aprender objetivos indicados de una manera razonablemente eficaz, entonces la tarea principal del profesor es hacer que los estudiantes se impliquen en actividades de aprendizaje que tienen la probabilidad de resultar en que logren esos objetivos" (pág. 429). Comprender y tener en cuenta las expectativas de los estudiantes es básico para conseguir que los estudiantes se impliquen en las actividades de aprendizaje.

El constructo de la "Confianza Académica" se está empleando para intentar comprender algunas diferencias entre las preferencias de los estudiantes en cuanto a la enseñanza y el aprendizaje (Sander y Sanders, 2003, ver cuadro 4). Las medidas de la confianza académica han demostrado que la confianza disminuye durante el primer curso de la universidad, y que disminuye más en aquellos estudiantes que predicen que probablemente no les irá tan bien como a los demás de su grupo. Hay muchas posibles explicaciones para esta bajada de confianza académica. Una explicación sería que los estudiantes entran en la universidad con mucha esperanza y buenas expectativas, que después las ven truncadas por un ambiente extraño, muy distinto de lo que habían experimentado en el instituto de Secundaria. Quizás estos alumnos encontraran un ambiente donde: (1) se les enseñaba de forma impersonal, tal vez en conferencias tradicionales con grupos muy grandes; (2) su acceso al profesorado era limitado y tal vez difícil de conseguir, y (3) las impresiones del profesor ante sus trabajos y exámenes eran limitadas y llegaban tarde.-

\section{Cuadro 4: Medida de la confianza en el estudio académico: un Informe- Resumen (Sander y Sanders, 2003)}

Guiado por el trabajo de Bandura sobre la auto-eficacia, este estudio pretendió determinar hasta qué punto las diferencias de expectativas de los estudiantes en cuanto a la enseñanza superior se podía explicar por distintos niveles de confianza.

Se construyó una escala de confianza académica (Academic Confidence Scale, ACS) que después se utilizó para encuestar a estudiantes del primer curso, explorando diferencias en confianza entre dos grupos muy distintos de estudiantes. Uno de los grupos también se encuestó sobre su confianza más adelante en el curso y al mismo tiempo; ellos rellenaron la Ladder of Aspiration (LofAsp, o sea, "escalera de aspiración”), para validar la Academic 
Confidence Scale. Con estos datos, se podía examinar con más profundidad la ACS, buscando factores subyacentes.

Análisis de factores de la ACS produjo seis factores (Estudio, Comprensión, Verbalización, Clarificación, Asistencia y Notas). La LofAsp proporcionó validación de la ACS. A partir de la LofAsp, se identificó un grupo pequeño que se calificaron como por debajo de la media nacional. Fue interesante este grupo, tanto en términos de sus puntuaciones ACS y su rendimiento académico. Las puntuaciones ACS mostraron una disminución significativa con el tiempo.

La comparación de la ACS entre los dos grupos de estudiantes sugiere que la confianza en muy poco puede ser responsable por las diferencias de expectativas de los estudiantes en cuanto a la enseñanza superior. La caída de puntuaciones ACS indica que la confianza académica se ve afectada por el rendimiento del estudiante, y no que afecta a ese rendimiento del estudiante. Los datos también plantean dudas sobre la capacidad de los estudiantes de pensar lógicamente con datos estadísticos así como sus perspectivas sobre su rendimiento más probable en el curso.

\section{Conclusiones}

Este trabajo expone argumentos a favor de intentar comprender a los estudiantes al principio de su educación universitaria. Cassidy y Eachus (2000) concluyen, “información de tipo perfil ... es beneficiosa tanto para informar la práctica de la enseñanza como para concienciar a los estudiantes en cuanto a las estrategias de aprendizaje y las habilidades de estudio" (p318). Información de tipo perfil que se recoge al principio del curso universitario puede proporcionar un punto de partida reconocido y claramente comprendido, a partir del cual se puede diseñar los ambientes de aprendizaje. Es necesario reconocer que los que alumnos que han terminado ahora la Secundaria van a necesitar que se trabaje con ellos (Laurillard, 1993; Northedge, 2003; Scott, 1999) para que adquieran habilidades y confianza en sus habilidades, para que lleguen a ser aprendices independientes, reflexivos, profundos y activos. La transición de la secundaria a la universidad exige mucho de los nuevos universitarios (Chemers, $\mathrm{Hu}$ y García, 2001; Cook y Leckey, 1999; Delaney, 2002; Lawrence, 2001; Lowe y Cook, 2003) y puede que la cultura en la que se encuentran los universitarios les ponga difícil a muchos que empiecen su carrera de forma eficaz, sobre todo a los que vienen de trasfondos tradicionalmente menos asociados con el acceso a la enseñanza superior.

A pesar del hecho de que más estudiantes necesiten una atención individualizada para poder prosperar en el ambiente universitario, se hace difícil que los profesores universitarios trabajen de forma eficaz con estudiantes individuales --de la manera que puede trabajar un 
profesor de secundaria-- viendo el aumento en número y diversidad de alumnos, la falta de asignar recursos adecuados, y un ambiente duro de cambiar. Sin embargo, hacer un esfuerzo tal como se describe en este trabajo puede ser un comienzo que merece la pena.

\section{Reconocimiento}

1. Este trabajo no habría sido posible sin mis compañeros, Keith Stevenson, Lalage Sanders y Paul Naylor. Tampoco habría sido posible sin todos los estudiantes que dedicaron su tiempo a rellenar nuestros cuestionarios.

\section{Referencias}

Bell, J.D. (1998). Smaller = Better? State Legislatures Magazine, June. Consultado el 24 de marzo, 2004, de http://www.ncsl.org/programs/pubs/s16class.htm

Biggs, J. (1999) Teaching for Quality Learning at University. Buckingham: Society for Research into Higher Education and Open University Press.

Biggs, J., Kember, D., y Leung, D.Y. (2001). The revised two factor Study Process Questionnaire: R-SPQ-2F. British Journal of Educational Psychology, 63, 3-19.

Brown, G. (1993). Effective Teaching, in Ellis, R., Quality Assurance for University Teaching. Society for Research in Higher Education and Open University Press.

Cassidy, S. y Eachus, P. (2000). Learning Style, Academic Belief Systems, Self-report Student Proficiency and Academic Achievement in Higher Education. Educational Psychology, 20, 2, $307-320$.

Chemers, M.M., Hu, L. y Garcia, B.F. (2001). Academic Self-efficacy and First-Year College Student Performance and Adjustment. Journal of Educational Psychology, 93, 1, $55-64$.

Clarebout, G., Elen, J., Johnson, W.L., y Shaw, E. (2002). Animated pedagogical agents: An opportunity to be grasped? Journal of Educational Multimedia and Hypermedia, 11, 3, $267-286$. 
Clarebout, G., Elen, J., Luyten, L. y Bamps, H. (2002). Assessing Epistemological Beliefs: Schommer's Questionnaire Revisited. Educational research and Development, 7, 1, $53-77$.

Clarebout, G., Lowyck, J. y Elen, J. (2003). Students' conceptions and "guided independent learning". Paper to be presented at the $10^{\text {th }}$ biennial conference for European Association for Research on Learning and Instruction (Earli). Padova, Italy, August 26-30.

Cook, A. y Leckey, J., (1999.) 'Do Expectations Meet Reality? A survey of changes in firstyear student opinion'. Journal of Further and Higher Education 23, (2): 57-171.

Delaney, A. M. (2002). Enhancing support for student diversity through research. Tertiary Education and Management, 8, 2, 145-166.

Davis, M. (2003). Barriers to Reflective Practice. Active Learning in Higher Education, 4, 3, 243-255.

Elen, J. y Clarebout, G. (2002). An invasion in the classroom: Influence of an ill-structured innovation on instructional and epistemological beliefs. Learning Environments Research, 4, 1, $87-105$.

Eurydice, 2000. Two Decades of Reform in Higher Education in Europe: 1980 onwards. Brussels: The Information Network on Education in Europe, Eurydice. Consultado el 24 de marzo, 2004, de http://www.eees.ua.es/otros documentos/towards a coherent.pdf

Field, J. (2002). Citezenship, Euaropean Enlargement and Higher Education: A Critical Perspective. Consultado el 24 de marzo, 2004, de http://www.srhe.ac.uk/Hern/eForum/Leiden/HERN-Leiden-UK-Field-text.pdf

Hart, D, (1999). Warning over school class sizes. Consultado el 24 de marzo, 2004, de http://news.bbc.co.uk/1/hi/education/specials/unions99/360299.stm

Hartley, J. (1998). Learning and Studying: A research perspective. London: Routledge.

HEQC (1995). What Are Graduates? Clarifying the Attributes Of 'Graduateness'. The Higher Education Quality Council (HEQC): Quality Enhancement Group (1995). Consultado el 24 de marzo, de http://www.lgu.ac.uk/deliberations/graduates/starter.html

Hill, F. (1995). 'Managing service quality in higher education: the role of the student as primary consumer'. Quality Assurance in Education 3 (3): 10-21. 
Kember, D., Leung, D., Jones, A., Loke, A.Y., McKay, J., Sinclair, K., Tse, H., Webb, C., Wong, F.K., Wong, M., y Yeung, E. (2000). Development of a questionnaire to measure the level of reflective thinking. Assessment and Evaluation in Higher Education, $25,381-389$

Jolliffe A, Ritter J, y Stevens D (2001) The On-Line Learning Handbook. London: Kogan Page.

Laurillard, D. (1993). Rethinking University Teaching: a framework for the effective use of educational technology. London: Routledge.

Lawrence, J., (2001). Academics and first year students: collaborating to access success in an unfamiliar university culture. Widening Participation and Lifelong learning, 3, 3, 4-14.

Leung, D. y Kember D. (2003). The Relationship Between Approaches to Learning and Reflection Upon Practice. Educational Psychology, 23, 1, 61 - 71.

Lowe, H. y Cook, A., (2003). Mind the Gap; are students prepared for higher education? Journal of Further and Higher Education, 27, 1, 53-76.

Learning Teaching Support Network (LTSN, 2002). Personal Development Planning. Consultado el 24 de marzo, 2004, de

http://www.ltsn.ac.uk/application.asp?app=resources.asp\&process=full record\&section =generic\&id $=66$

Mann, S, (2001). Alternative Perspectives on the Student Experience: alienation and engagement. Studies in Higher Education, 26, 1, 7 - 19.

Maunder, R.E. y Harrop, A. (2003). Investigating Students' Perceptions of What Contributes to Productive Seminars and Lectures and Staff Predictions of Students' Perceptions: how well do staff know their students? Journal of Further and Higher Education, 27, 4, 443-456. 
Ministerio de Educación, Cultura y Deporte, España. Seguir enlaces: (i) Evolución de alumnos matriculados y titularidad de la universidad and (ii) Datos sociológicos de los alumnos matriculados. Consultado el 24 de marzo, 2004, de http://wwwn.mec.es/educa/ccuniv/html/estadistica/series/index.html

Mora, J. G., (1999). Two decades of change in Spanish higher education. International Higher Education, Fall, 1999. Consultado el 24 de marzo, 2004, de http://www.bc.edu/bc_org/avp/soe/cihe/newsletter/News17/text12.html

Natfhe (referencia de internet). Opening Up Higher Education: new investment for a new higher education. Consultado el 24 de marzo, 2004, de http://www.natfhe.org.uk/down/csrmaker.pdf

Northedge, A. (2003). Rethinking Teaching in the Context of Diversity. Teaching in Higher Education, 8, 1, $17-32$.

Sander, P, (2002). Group work with students. LTSN Psychology Teaching Tips. Consultado el 24 de marzo, 2004, de http://ltsnpsy.york.ac.uk/LTSNAsp/tipsbytopictest.asp?Field=IDteachingproblem\&searc $\underline{\text { hfor=Large+groups }}$

Sander, P, y Stevenson, K. (2002). Why we don't like student presentations: the students speak. The Psychologist in Wales (Newsletter of the Welsh Branch of the British Psychological Society), 14, 3-12.

Sander, P., Stevenson, K., King, M. y Coates, D. (2000). University Students' Expectations of Teaching. Studies in Higher Education, 25, 3, pages 309 - 323

Sander, P. y Sanders, L. (2003). Measuring confidence in academic study: A summary report. Electronic Journal of research in Educational Psychology and Psychopedagogy, 1, 1. Consultado el 24 de marzo, 2004, de http://www.investigacionpsicopedagogica.org/revista/articulos/1/english/Art 1_1.pdf

Sander, P. y Sanders, L. (2005). Giving presentations: the impact on students' perceptions. Psychology Teaching Review, 11, 1, 25-39.

Sander, P., Sanders, L. y Stevenson, K. (2002). Engaging the Learner: Reflections on the use of Student Presentations. Psychology Teaching Review, 76-89.

Schwartz, P., Mennin, S. y Webb, G. (2001). Problem-Based Learning: Case studies, experience and practice. London, Kogan Page. 
Schommer, M. (1990). Effects of beliefs about the nature of knowledge on comprehension. Journal of Educational Psychology, 82, 3, 498 - 504.

Schommer, M. (1998). The influence of age and schooling on epistemological beliefs. The British Journal of Educational Psychology, 68, 551-562.

Scott, S. V. (1999). 'The Academic as a Service provider; is the customer 'always right'?' Journal of Higher Education Policy and Management, 21 (2): 193-202.

Schuell, T.J. (1986). Cognitive Conceptions of Learning. Review of Educational Research, $56,4,411-436$.

Stevenson, K. y Sander, P. (2002). Medical students are from Mars - business and psychology students are from Venus - University lecturers are from Pluto. Medical Teacher, $24,1,27-31$.

Stevenson K., Sander P. y Naylor P. (1996) Student perceptions of the tutor's role in distance learning, Open Learning, 11, 1, 22-30

Stevenson, K., Sander, P. y Naylor, P. (1997). ELPO - A Model that uses Student Feedback to Develop Effective Open Tutoring, Open Learning, 12, 2, 54-59.

Thorne, E.M. y Cuthbert, R. (1996). Autonomy, Bureaucracy and Competition: The ABC of Control in Higher Education, en: R. Cuthbert (Ed), Working in Higher Education. Buckingham, Society for Research into Higher Education and Open University Press.

UWIC, (referencia de internet). Learning and Teaching Strategy. Consultado el 24 de marzo, 2004, de http://www.uwic.ac.uk/ltsu/documents/L\&T Strategy 2nd Draft + Targets - July 2000 WEB VERSION.doc

Winn, S., (2002). Student Motivation: a socio-economic perspective. Studies in Higher Education, 27, 4, 445-457 